\title{
Simultaneous determination of purine metabolites in ovine urine and blood plasma by high-performance liquid chromatography
}

\author{
M. Czauderna ${ }^{1}$ and J. Kowalczyk \\ The Kielanowski Institute of Animal Physiology and Nutrition, \\ Polish Academy of Sciences \\ 05-110 Jabłonna, Poland
}

\begin{abstract}
High-performance liquid chromatographic methods for directly determining products of nucleic acid and purine catabolism in urine and blood plasma of sheep were developed. Urine and plasma samples were diluted 1:3 with deionized water. Fractionation and quantification of allantoin, uric acid, hypoxanthine and xanthine were performed using two Nova-Pak $\mathrm{C}_{18}$ columns $(300 \times 3.9 \mathrm{~mm}$, Waters). Binary gradient programs and UV detection were used for purine metabolite analysis. Satisfactory fractionation of all analytes in urine and plasma was obtained in less than 19 and 21 min, respectively. The average recoveries of standard compounds added to the assayed samples were $\sim 100 \%$. The low coefficient of variation $(1-2 \%)$ as well as the low detection limits $(0.09-0.36$ $\mathrm{nmol}$ ) indicate satisfactory precision, reproducibility and sensitivity of the proposed methods. These chromatographic methods are suitable for routine quantification of purine metabolites in a large number of samples.
\end{abstract}

KEY WORDS: purine metabolites, sheep, urine, blood, liquid chromatography

\section{INTRODUCTION}

In ruminants, dietary nucleic acids are degraded in the rumen in a series of reactions to form allantoin and oxypurines (i.e. uric acid, hypoxanthine, and xanthine) in blood plasma and urine. Allantoin excreted in the urine is the main end product of purine metabolism (Chen et al., 1996; Kowalczyk et al., 2003). It originates from purine bases of rumen microorganisms, feed purines, and endogenous purines from tissue turnover. Fortunately, in sheep and other ruminants there may be a close relationship between the production of microbial protein in the rumen and the excretion of purine

\footnotetext{
${ }^{1}$ Corresponding author: e-mail: m.czauderna@ifzz.pan.pl
} 
metabolites (PM) in urine and plasma. This has led to renewed interest in the accurate quantification of PM in urine and plasma as a possible indicator of the amount of microbial protein entering the small intestine. Considering the above, it is essential to develop simple, accurate, and precise analytical methods using high-resolution reversed-phase HPLC and selective photodiode array detection.

\section{MATERIAL AND METHODS}

Allantoin, uric acid, hypoxanthine, and xanthine (PM) were obtained from Sigma (USA), while HPLC-grade acetonitrile was purchased from Lab-Scan (Ireland). Other chemicals were of analytical reagent grade and purchased from POCh (Poland). HPLC-grade water was prepared using a Milli-Q system (Millipore, Canada). Eluent A was prepared from $0.0025 \mathrm{M} \mathrm{NH}_{4} \mathrm{H}_{2} \mathrm{PO}_{4}$ buffered to $\mathrm{pH} 3.5$ with $10 \%$ phosphoric acid. Eluent B was acetonitrile. Eluent A was filtered through a $0.45 \mu \mathrm{m}$ membrane filter. The HPLC instrument used consisted of a Waters 625LC system, which includes a controller for gradient elution, two Waters 515 pumps, a Waters 996 photodiode array detector (PAD), a Waters ${ }^{\mathrm{TM}}$ 717plus WISP autosampler, and a Waters in-line degasser AF. Development of the analytical methods was performed using Millennium ${ }^{32}$ software (v. 4.0). The PAD was operated in a UV range from 190 to $350 \mathrm{~nm}$ with a spectral resolution of $1.2 \mathrm{~nm}$ and a measurement frequency of 1 spectrum per sec. The columns used were two Nova-Pak columns $(4 \mu \mathrm{m}, 300 \times 3.9 \mathrm{~mm}$, Waters $)$ in conjunction with a guard column. Binary gradient programs (Table 1) were used for separation of PM in urine and blood plasma (Czauderna and Kowalczyk, 2000).

Table 1. Binary gradient programs used for analysis of purine metabolites in ovine urine (Program A) and blood plasma (Program B) (separations were performed at a column temperature of $26^{\circ} \mathrm{C}$ )

\begin{tabular}{|c|c|c|c|c|c|c|c|}
\hline \multicolumn{4}{|c|}{ Gradient program A (ovine urine) } & \multicolumn{4}{|c|}{ Gradient program B (plasma) } \\
\hline time & flow-rate & solvent A & solvent B & time & flow-rate & solvent $\mathrm{A}$ & solvent B \\
\hline $\min$ & $\mathrm{ml} \mathrm{min} \min ^{-1}$ & $\%$ & $\%$ & $\min$ & $\mathrm{ml} \mathrm{min}-1$ & $\%$ & $\%$ \\
\hline 0 & 0.9 & 100 & 0 & 0 & 0.8 & 100 & 0 \\
\hline 9.5 & 0.9 & 100 & 0 & 7.0 & 0.75 & 100 & 0 \\
\hline 10.0 & 0.9 & 99.5 & 0.5 & 20.0 & 0.9 & 100 & 0 \\
\hline 20.5 & 0.9 & 90 & 10 & 20.8 & 0.9 & 5 & 95 \\
\hline 20.8 & 1.0 & 5 & 95 & 30.0 & 1.7 & 5 & 95 \\
\hline 26.0 & 1.4 & 5 & 95 & 35.5 & 1.7 & 5 & 95 \\
\hline 32.0 & 1.7 & 5 & 95 & 35.8 & 1.1 & 100 & 0 \\
\hline 33.0 & 1.1 & 100 & 0 & 39.0 & 1.0 & 100 & 0 \\
\hline 60 & 0.9 & 100 & 0 & 70 & 0.9 & 100 & 0 \\
\hline
\end{tabular}

Ovine urine samples were diluted 1:3 with water. The resulting solution was immediately analysed using gradient program $\mathrm{A}$ or stored at $-20^{\circ} \mathrm{C}$ when not in use. Blood samples were collected in heparinized tubes and immediately centrifuged 
at $1500 \mathrm{~g}$ for $20 \mathrm{~min}$ at $\sim 4^{\circ} \mathrm{C}$ (Czauderna and Kowalczyk, 1997). The obtained plasma samples were diluted 1:3 with water and then immediately injected onto an HPLC column. Injection volumes were 15-25 $\mu 1$.

\section{RESULTS}

The major analytical problem in the current study was obtaining satisfactory separation of PM. Plasma and urine samples contain endogenous components with a very similar polarity and a strong chromophore in the UV wave-length range, which coincide in particular with the UV spectra of allantoin and uric acid. Unfortunately, allantoin, like many other endogenous species, is poorly retained on $\mathrm{C}_{18}$ columns. Therefore, to avoid problems due to overlapping peaks, two long $\mathrm{C}_{18}$ columns together with photodiode monitoring were used. Our current study documented that in urine and plasma samples, the optimal peak resolution between allantoin and the nearest interfering species (i.e. noises) was obtained when the buffer was adjusted to $\mathrm{pH} 3.5$ and allantoin in effluents was monitored at $215 \mathrm{~nm}$. As can be seen from detailed chromatographic studies of urine and plasma samples, in gradient program $\mathrm{A}$, the allantoin peak (in urine samples) eluted at $5.4 \pm 0.1 \mathrm{~min}$, while in gradient program B (in plasma samples) it eluted at $6.1 \pm 0.1 \mathrm{~min}$. Satisfactory fractionation and quantification of oxypurines (i.e. uric acid, hypoxanthine and xanthine) from background fluctuation and interfering species in urine and freshly prepared plasma samples was obtained using gradient programs $\mathrm{A}$ and $\mathrm{B}$, and UV monitoring at longer wavelengths (Table 2). No purine metabolite peaks were present in the blank when the both gradient programs A and B were applied.

Table 2. Detection of purine metabolites in urine (Program A) and blood plasma (Program B)

\begin{tabular}{lcccc}
\hline \multirow{2}{*}{$\begin{array}{l}\text { Purine } \\
\text { metabolite }\end{array}$} & \multirow{2}{*}{$\begin{array}{c}\text { Wave length } \\
\text { detection, } \mathrm{nm}\end{array}$} & $\begin{array}{c}\text { Detection limit } \\
\mathrm{nmol}\end{array}$ & & \multicolumn{2}{c}{ Retention time, min } \\
\cline { 4 - 5 } & & & $\mathrm{A}$ & $\mathrm{B}$ \\
\hline Allantoin & 215 & 0.09 & $5.4 \pm 0.1$ & $6.1 \pm 0.1$ \\
Uric acid & 285 & 0.11 & $13.6 \pm 0.1$ & $15.7 \pm 0.2$ \\
Hypoxanthine & 250 & 0.12 & $15.7 \pm 0.2$ & $17.6 \pm 0.2$ \\
Xanthine & 267 & 0.36 & $17.5 \pm 0.2$ & $20.7 \pm 0.2$ \\
\hline
\end{tabular}

The precision of the presented methods was assessed by performing replicate injections of freshly processed urine and plasma samples. Low variation coefficients $(1-2 \%)$ for quantification of PM in urine and plasma samples were obtained using the proposed UV monitoring at longer wavelengths (Table 2). The recoveries of PM added to urine and plasma samples were between 96 and 102\%. The accuracy of the HPLC methods was also examined using detection of PM at various UV 
wavelengths $(205,210,225$ and $231 \mathrm{~nm})$, buffers adjusted to $\mathrm{pH} 2.5,4$ and 6 , and using more acetonitrile from minutes 8 to 20 of chromatographic runs. Fortunately, no additional endogenous species with overlapping analytical peaks were found using modified chromatographic conditions and non-optimal UV detection modes.

\section{DISCUSSION}

Comparison of the obtained UV spectra of PM in urine and plasma with the UV spectra of PM in standards (data not presented) indicated that all peaks corresponding to PM are pure $(\sim 100 \%)$ and free from the close presence of un-identified species. Thus, all analytical peaks can be integrated using the total peak method as devoid of substantial co-eluting endogenous impurities with peaks absorbing in the applied UV detection range. This makes it possible to obtain satisfactory reproducibility of the presented HPLC methods. These procedures are more sensitive ( $\sim 2$-fold) for PM analysis compared with our previous methods (Czauderna and Kowalczyk, 1997, 2000) since urine and blood samples are less diluted.

\section{CONCLUSIONS}

The presented HPLC methods enable very rapid and simple determination of PM in urine and blood plasma. Since assayed samples are only simply diluted with water, the current methods avoid problems associated with pre- or postcolumn derivatization as well as with using an internal standard. The described methods provide a sensitive, accurate, and precise non-invasive analytical tool for estimation of ruminal microbial protein supply to sheep. This approach only requires collection of urine and blood. The HPLC method using gradient program $\mathrm{B}$ can be applied to analysis of allantoin levels in ruminal digesta.

\section{REFERENCES}

Chen X.P., Samaraweera L., Kyle D.J., Ørskov E.R., Abeygunawardene H., 1996. Urinary excretion of purine derivatives and tissue xanthine oxidase (EC 1.2.3.2) activity in buffaloes (Bubalis bubalis) with special references between buffaloes and Bos taurus cattle. Brit. J. Nutr. 75, 397-407

Czauderna M., Kowalczyk J., 1997. Simultaneous measurement of allantoin, uric acid, xanthine and hypoxanthine in blood by high-performance liquid chromatography. J. Chromatogr. B 704, 89-98

Czauderna M., Kowalczyk J., 2000. Quantification of allantoin, uric acid, xanthine and hypoxanthine in ovine urine by high-performance liquid chromatography and photodiode array detection. J. Chromatogr. B 744, 129-138

Kowalczyk J., Czauderna M., Pająk J.J., Strzetelski J.A., Skomiał J., 2003. Recovery in urine of allantoin administered intravenously at differentiated levels of feeding sheep. In: Progress in Research on Energy and Protein Metabolism, Rostock (Germany). EAAP Publication, No. 109, pp. 773-776 